\title{
In vitro effects of (+)MK-801 (dizocilpine) and memantine on $\beta$-amyloid peptides linked to
}

\section{Alzheimer's Disease}

Susan E. Coombs ${ }^{a, c}$, Sudeep Banjade ${ }^{b}$, Ksenia Kriksunov ${ }^{c}$, Nicolina Clemented, Jing Zhao ${ }^{d}$, Chunyu Wang ${ }^{d}$, Richard E. Gillilan ${ }^{e}$, and Robert E. Oswald ${ }^{*}$

${ }^{\mathrm{a}}$ Molecular Medicine, ${ }^{\mathrm{b}} \mathrm{Weill}$ Institute for Cell and Molecular Biology, ${ }^{\mathrm{c}}$ Molecular Biology and Genetics, ${ }^{e}$ MacCHESS (Macromolecular Diffraction Facility at CHESS), Cornell University, Ithaca, NY 14853, U.S.A.

${ }^{\mathrm{d}}$ Department of Biological Sciences and Center of Biotechnology and Interdisciplinary Studies, Rensselaer Polytechnic Institute, Troy, NY 12180, U.S.A.

\section{Corresponding Author}

*Robert E. Oswald, Molecular Medicine, Cornell University, Ithaca, NY 14853, U.S.A. reo1@cornell.edu (OrcidID 0000-0003-3028-7922) 


\section{Supplemental Information}

\section{MATERIALS AND METHODS}

MATERIALS. All materials used were commercially available. SensoLyte ${ }^{\circledR}$ ThT $\beta$-Amyloid [142] and [1-40] Aggregation kits were purchased from AnaSpec (Fremont, CA). A $\beta$ peptides (synthetically produced via solid phase peptide synthesis and provided as a lyophilized powder soluble in $\mathrm{NH}_{4} \mathrm{OH}$ ), ThT, and Tris-based neutral assay buffers were supplied with the kit. ${ }^{15} \mathrm{~N}$ labeled $A \beta[1-40]$ and $A \beta[1-42]$ peptides were purchased from rPeptide (Watkinsville, GA) and $\mathrm{A} \beta[1-42]$ peptides with ${ }^{13} \mathrm{C}$ and ${ }^{15} \mathrm{~N}$ labeling on the arginine at position 5 and on lysines at positions 16 and 28 were purchased from AnaSpec. Fluorescence measurements were made in Greiner non-binding, black 96-well plates with flat bottoms that were purchased from E \& K Scientific (Santa Clara, CA). Protein Lo-Bind (PCR Clean) tubes purchased from Eppendorf (Hauppauge, NY) were used for all peptide solutions. (+)MK-801 hydrogen maleate (dizocilpine), and memantine hydrochloride were purchased from Sigma Aldrich (St. Louis, MO), deuterium oxide and NMR tubes from Sigma-Aldrich, and Formvar/Carbon 200 mesh Copper microscope grids were from Electron Microscopy Sciences (Hatfield, PA). Hellmanex II was purchased from Hellma USA Inc. (Plainview, NY). All other chemicals used were reagent grade and purchased from Sigma-Aldrich. Water from a BarnsteadTM MicroPureTM Water Purification System was used to prepare solutions.

THIOFLAVIN T ASSAYS. The assays were run as described in the AnaSpec protocol. The preparation included hydration of the peptide in the buffer for three $(\mathrm{A} \beta[1-40])$ or five $(\mathrm{A} \beta[1$ 42]) minutes followed by centrifugation at 10,000 r.p.m. for 5 min. By visual inspection, the solution was clear at this point. The ThT concentration was $0.2 \mathrm{mM}$ in a buffer of $50-\mathrm{mM}$ Tris, 
150-mM NaCl, $\mathrm{pH}$ 7.2. The peptide had been lyophilized from a solution of ammonium hydroxide, acetonitrile and water. ${ }^{1}$ Ammonium hydroxide predisposes the peptide towards a lack of aggregates in the initial solution; ${ }^{1}$ however, the BioSAXS studies suggested that at least some aggregation was present by 15 min after the preparation of the sample. The lack of an observable lag phase is likely attributable to a small amount of aggregation in the original solution and the time lag for the start of each experiment. Several experiments were done with peptide from rPeptide, which did show a lag phase but had otherwise qualitatively similar results [dramatic decrease in ThT signal at later times in the presence of $(+) \mathrm{MK}-801]$. These preparations were somewhat less reproducible in the ThT assay than the preparation (AnaSpec) used for the bulk of the experiments. Three to six replicates of each experimental condition were used in each assay in Greiner 96-well plates, and the experiments were repeated 11 times, with minor variations. The plate was inserted into a Molecular Devices Spectromax M2 reader (within 10 minutes of preparation), where the excitation wavelength had been set to $440 \mathrm{~nm}$ and the emission wavelength to $485 \mathrm{~nm}$. Fluorescence values were recorded every 5 minutes, with orbital shaking for 15 seconds prior to each reading. Readings were continued for an initial period of 3 hours, and then repeated at various times over the next 24 hours. The temperature was $\sim 25^{\circ} \mathrm{C}$ and varied less than one degree centigrade. The $\mathrm{pH}$ of the solution did not change during the course of an experiment. When there was a pause between the 3-hr reading periods (e.g. Figure 1B), the plate was shaken on a New Brunswick G10 gyratory orbital shaker at 100 r.p.m. at ambient temperature. During this time, to limit evaporation and to avoid unnecessary exposure to light, the plate was covered and sealed with a Parafilm ${ }^{\mathrm{TM}}$ sheet, then wrapped in aluminum foil, sealed in a Ziplock ${ }^{\mathrm{TM}}$ plastic bag and taped shut. 
TRANSMISSION ELECTRON MICROSCOPY. Two independent ThT assays of A $\beta[1-42]$ in the presence or absence of $(+) \mathrm{MK}-801$ or memantine were run in parallel with a duplicate set of wells except that the ThT was replaced by an equal volume of assay buffer. At given time points, $10 \mu \mathrm{L}$ were removed from each well that did not contain ThT. The plate was then returned to the plate reader and fluorescence readings continued. The $10-\mu \mathrm{L}$ samples were applied to a Formvar/Carbon 200 mesh Copper microscope grid; after 10 minutes excess liquid was removed with filter paper. The peptides were then stained with filtered $2 \%$ ammonium molybdate for 1 minute, and imaged at $60 \mathrm{kV}$ on an FEI Morgnani 268 transmission electron microscope (Department of Molecular Biology and Genetics, Cornell University).

NMR SPECTROSCOPY. Two-dimensional NMR spectroscopy of the monomeric species. ${ }^{15} \mathrm{~N},{ }^{1} \mathrm{H}$ HSQC spectra of ${ }^{15} \mathrm{~N}$-labeled $\mathrm{A} \beta[1-40]$ and [1-42] from rPeptide, in the presence or absence of (+)MK-801, were recorded at $277 \mathrm{~K}$ on a Bruker $800 \mathrm{MHz}(18.8$ Tesla) instrument at the NMR core facility at Rensselaer Polytechnic Institute. The peptide concentration was $50 \mu \mathrm{M}$ and, when present, the concentration of (+)MK-801 was $2 \mathrm{mM}$. Several lower concentrations of $(+)$ MK-801 were used in the experiment with $A \beta[1-40]$, with no observed difference in the spectra.

One-dimensional NMR spectroscopy. A $0.25 \mathrm{mg}$ sample of $A \beta[1-42]$ peptide from AnaSpec was dissolved in $195 \mu \mathrm{L}$ of the ThT assay buffer [with or without $2.5 \mathrm{mM}(+) \mathrm{MK}-801$ ] with $25 \mu \mathrm{L}$ of $\mathrm{D}_{2} \mathrm{O}(250-\mu \mathrm{M}$ peptide). The reaction mixture was transferred to a Shigemi NMR tube and placed in a Varian Inova 500-MHz spectrometer (11.7 Tesla) (Department of Molecular Medicine, Cornell University) (within 10 minutes of preparation). One-dimensional proton spectra were recorded for 22 hours (256 transients were averaged at $5.37 \mathrm{~min}$. intervals). Methyl peaks were 
integrated and normalized to the first point. In some experiments, after 1 hour $30 \mu \mathrm{L}$ of $2.5-\mathrm{mM}$ (+)MK-801 were added to the reaction mixture without the ligand in the NMR tube, which was then returned to the spectrometer and recording was resumed.

BIOSAXS. A $\beta[1-42]$ peptides from AnaSpec and from rPeptide were prepared in a manner identical to that used in the Thioflavin $\mathrm{T}$ assays (with the exception that Thioflavin $\mathrm{T}$ was not added). Samples were centrifuged at 10,000 r.p.m. for 5 min. immediately before data acquisition. $30 \mu \mathrm{L}$ samples were loaded into a capillary flow cell at $22^{\circ} \mathrm{C}$ and oscillated during exposure to the X-ray beam to reduce potential radiation damage. Between each sample, the capillary cell was washed and dried using the following protocol: water, $2 \% \mathrm{v} / \mathrm{v}$ Hellmanex II in $10 \% \mathrm{v} / \mathrm{v}$ ethanol, water, $100 \%$ methanol, then dry nitrogen. All SAXS experiments were collected at the Cornell High-Energy Synchrotron Source (CHESS) using the ID7A BioSAXS/HP-Bio beamline. ${ }^{2,3}$ Scattering patterns were measured using an EIGER 4M detector mounted in vacuo (Dectris, Switzerland) and normalized using transmitted direct beam intensity measured at the beamstop. With an X-ray energy of $9.928 \mathrm{keV}(1.249 \AA)$ and a sample-todetector distance of $1707 \mathrm{~mm}$, scattering profiles covered a q-range from $0.0084 \AA^{-1}-0.5348 \AA^{-}$ ${ }^{1}$. The $250 \mu \mathrm{m} \times 250 \mu \mathrm{m} X$-ray beam was attenuated to approximately $10^{12}$ photons $/ \mathrm{s}$ to reduce the potential for damage during repeated exposures. For each time measurement, $10 \mathrm{X} 1 \mathrm{sec}$ exposures were taken with the resulting scattering profiles being compared for similarity using the CorMap criterion with a P-value threshold of $0.01{ }^{4}$ Because the exposed sample volume of $\sim 0.1 \mu \mathrm{L}$ was small in comparison to the $30-\mu \mathrm{L}$ total sample volume, damaged material is expected to diffuse out of the beam path, given enough time. The characteristic diffusion time scale, $t$, for an initial point of sample to spread to $\sigma$ width is given by $t=\sigma^{2} /(2 \mathrm{D})$ where $\mathrm{D}$ is the sample diffusion constant. For a small protein (lysozyme) $\mathrm{D}=10^{6} \mathrm{~cm}^{2} / \mathrm{s}$, and this translates into $\mathrm{t}$ 
$\sim 5$ min. With this strategy in mind, we collected time measurements every $15 \mathrm{~min}$. for up to three hours. At the end of the time series, an identical sample was placed in the capillary cell and an additional data set was collected to test, by comparison of the data, for accumulated radiation damage in the original sample. In the presence of $(+) \mathrm{MK}-801$, data collection was terminated due to precipitation visually observed in the capillary tube. This was manifested in the scattering profiles by statistically significant differences between the ten replicate profiles (as judged by the CorMap criterion). Background subtraction of SAXS buffer and further data and statistical analyses were performed using the free open-source software, RAW. ${ }^{5}$ The GNOM program from the ATSAS suite ${ }^{6}$ was used to determine $\mathrm{P}(\mathrm{r})$ plots. Unique three-dimensional shapes could not be determined from these scattering profiles as determined by AMBIMETER (ATSAS), probably due to the high level of polydispersity.

\section{MODELING THIOFLAVIN T ASSAYS}

In the absence of drug, the rise in fluorescence in the ThT assay can be described as a twoexponential rise in signal. Although a double exponential does not have a direct mechanistic interpretation, the model given in the Supplemental Information, based on a growing chain of oligomers (Scheme S1), can also fit the data well (Figure S1). However, the interesting timecourse is that with (+)MK-801 and memantine. The initial portion of the data initially follows a similar double-exponential time course (also modeled by the growing chain of oligomers) but it is followed by a precipitous decline. The initial attempts to model this were simply transitions to a linear chain of states (Scheme S1), with the last state nonfluorescent and absorbing. The rate equations were solved as described previously ${ }^{7}$ using MatLab. ${ }^{8}$ No satisfactory fits could be obtained, presumably because of the abrupt nature of the decline in fluorescence. However, Scheme S1 could be used to model the rise in fluorescence with only one rate constant (Runge- 
Kutta $^{9}$ ), and, at a given time, the loss in fluorescence could be characterized by a single exponential decline. Using this approach, the time course could be reproduced. While a unique mechanism cannot be obtained from these rather sparse data with limited dynamic range, perhaps more qualitative conclusions can be drawn. First of all, the aggregation steps in the presence of (+)MK-801 or memantine have higher rates than those in its absence. The decline in fluorescence, which appears to be associated with an insoluble aggregate, is not a result of the precipitation of an aggregate of a unique size. That is, the oligomer does not grow to a given size and then fall out of solution. Rather, once the precipitation is triggered, it results in the exponential loss in signal of all soluble aggregates, presumably associated with precipitation. A time course similar to that in the fluorescence experiments was observed with BioSAXS in $(+)$ MK-801 samples. The pair distribution function indicated a growing particle size up to a point at which the $\mathrm{I}(0)$ increased dramatically followed by the inability to collect consistent data (i.e., the ten replicates were not consistent). As noted in the text, this was not due to radiation damage but rather precipitation of the peptide as was observed clearly in the sample cell. A similar phenomenon was not observed in the absence of (+)MK-801.

\section{Scheme S1:}

$$
\begin{gathered}
A+A \stackrel{k}{\rightarrow} A_{2}+A \stackrel{k}{\rightarrow} A_{3}+A \stackrel{k}{\rightarrow} A_{4}+A \stackrel{k}{\rightarrow} \cdots A_{n-1}+A \stackrel{k}{\rightarrow} A_{n} \\
A_{2}+A_{2} \stackrel{k}{\rightarrow} A_{4}+A_{2} \stackrel{k}{\rightarrow} A_{6}+A_{2} \stackrel{k}{\rightarrow} A_{8} \cdots A_{n-2}+A_{2} \stackrel{k}{\rightarrow} A_{n} \\
A_{3}+A_{2} \stackrel{k}{\rightarrow} A_{5}+A_{2} \stackrel{k}{\rightarrow} A_{7}+A_{2} \stackrel{k}{\rightarrow} A_{9} \cdots A_{n-2}+A_{2} \stackrel{k}{\rightarrow} A_{n} \\
A_{3}+A_{3} \stackrel{k}{\rightarrow} A_{6}+A_{3} \stackrel{k}{\rightarrow} A_{9}+A_{3} \stackrel{k}{\rightarrow} A_{12} \cdots A_{n-3}+A_{3} \stackrel{k}{\rightarrow} A_{n} \\
A_{4}+A_{3} \stackrel{k}{\rightarrow} A_{7}+A_{3} \stackrel{k}{\rightarrow} A_{10}+A_{3} \stackrel{k}{\rightarrow} A_{13} \cdots A_{n-3}+A_{3} \stackrel{k}{\rightarrow} A_{n}
\end{gathered}
$$


The rate equation describing this mechanism is:

$$
\frac{d A_{i}}{d t}=k \sum_{i}^{j-1} A_{i} A_{j-1}-k A_{j} \sum_{i=1}^{n} A_{i}
$$

Equation S1

The rates for each step do not necessarily have to be identical, but the data can be described adequately using only a single rate constant for each step. The rate equation (Equation S1) can be integrated using the Runge-Kutta method, ${ }^{9}$ and the rate constant and the number of states (n) fit to the data using a Simplex algorithm. ${ }^{9}$

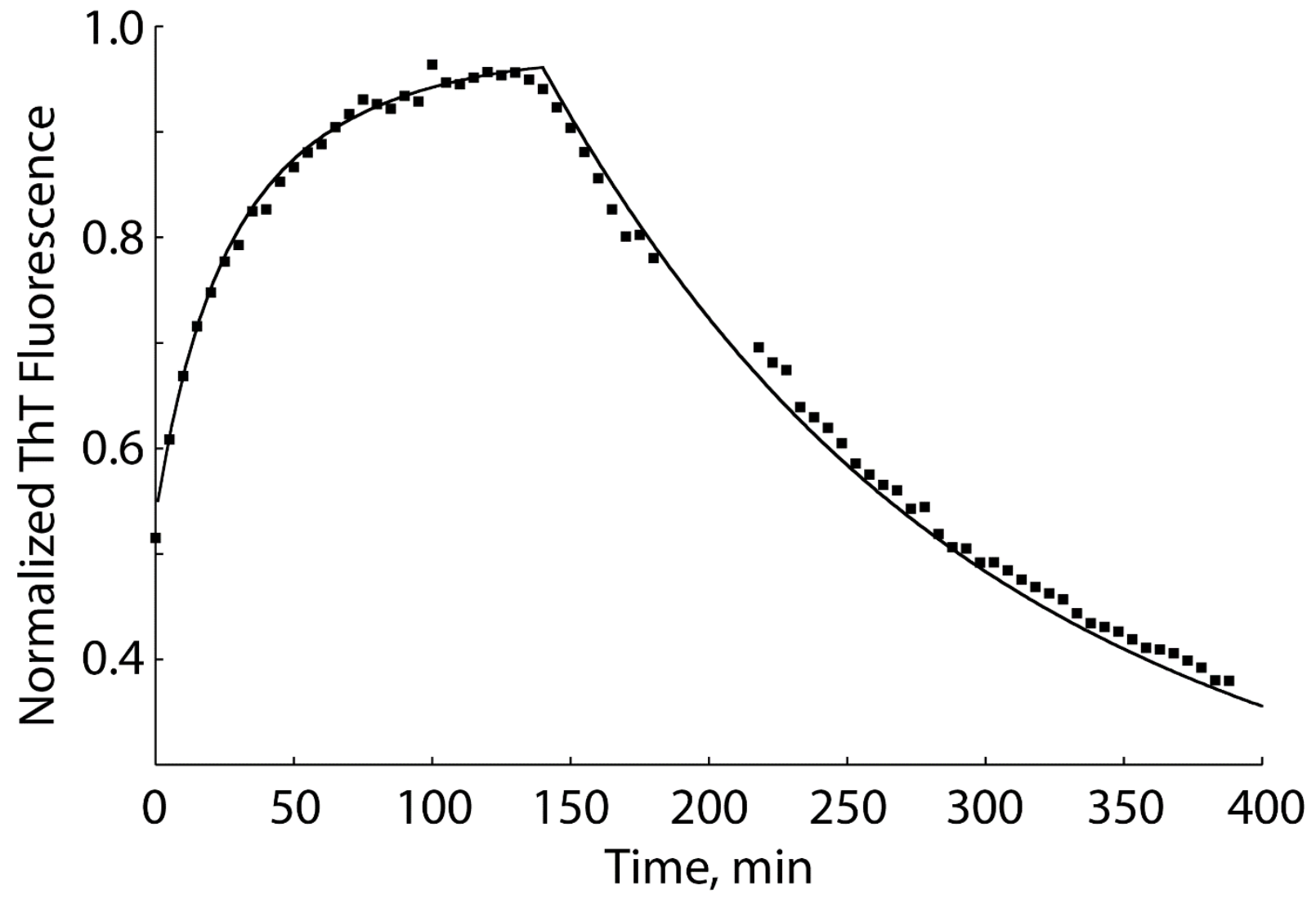


Figure S1: The data (solid squares) are from Figure 1A, 30- $\mu \mathrm{M} A \beta[1-42]$ with 2-mM (+)MK801. The fit, shown by the solid line, was done as described here in the Supplemental Information, with the rate constant of aggregation $(k)$ of $0.5 \mathrm{~min}^{-1}$, the total number of states of 80 , and the exponential decay rate constant of $0.02 \mathrm{~min}^{-1}$. The trigger point for the exponential decay was state 32 .

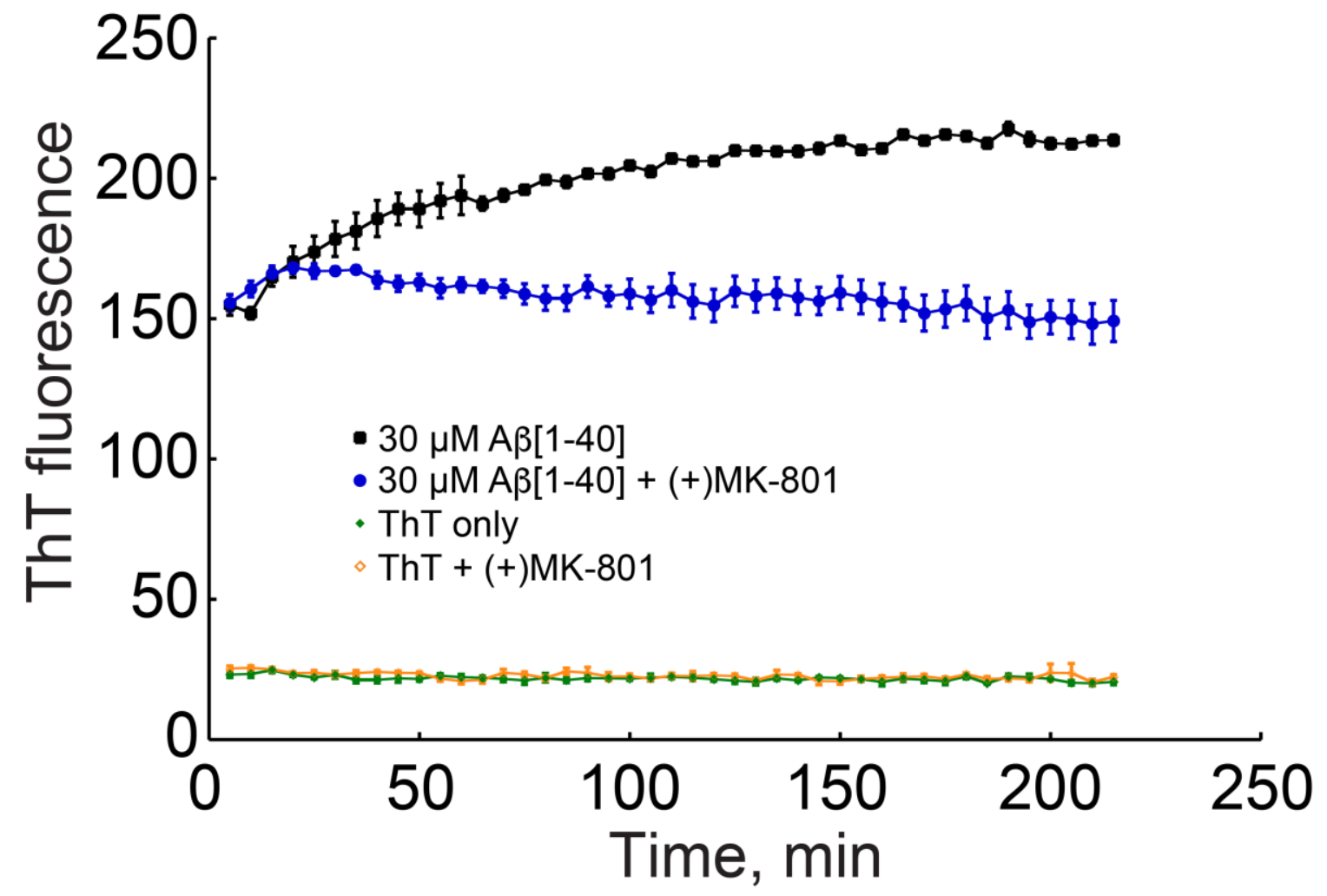

Figure S2: Formation of $\beta$-sheet structure by $30-\mu \mathrm{M} A \beta[1-40]$ in the presence and absence of 2$\mathrm{mM}(+) \mathrm{MK}-801$ measured using ThT fluorescence. Also shown is ThT alone and ThT with 2$\mathrm{mM}(+) \mathrm{MK}-801$ in the absence of $\mathrm{A} \beta[1-40]$. Error bars are the standard error of the mean. Fluorescence is shown as Arbitrary Units. 

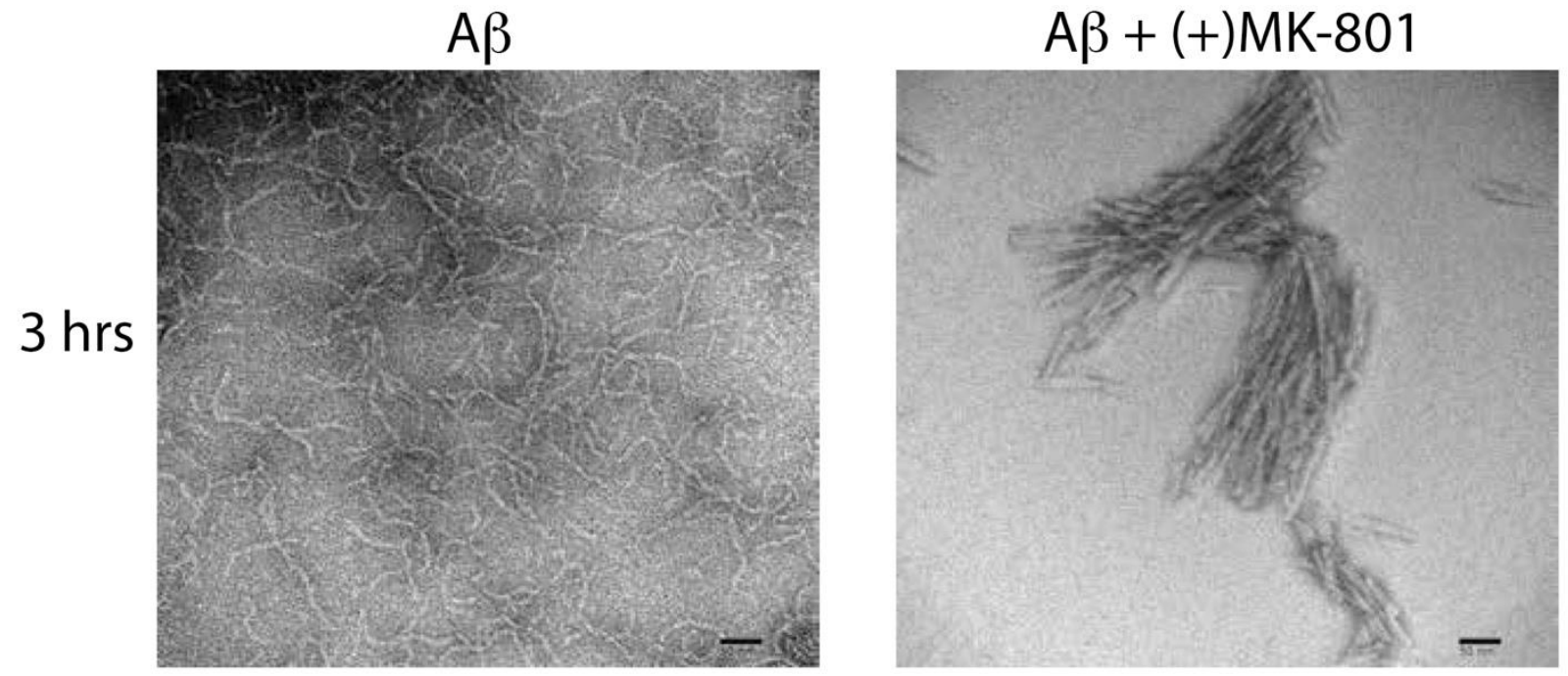

Figure S3: Electron micrographs of $25-\mu \mathrm{M} A \beta[1-42]$ in the presence or absence of 2-mM $(+)$ MK-801. The peptide was deposited on grids in assay buffer $(\mathrm{pH} 7.2)$ three hours after initiation of the experiment and stained with ammonium molybdate. Scale bar $50 \mathrm{~nm}$. 


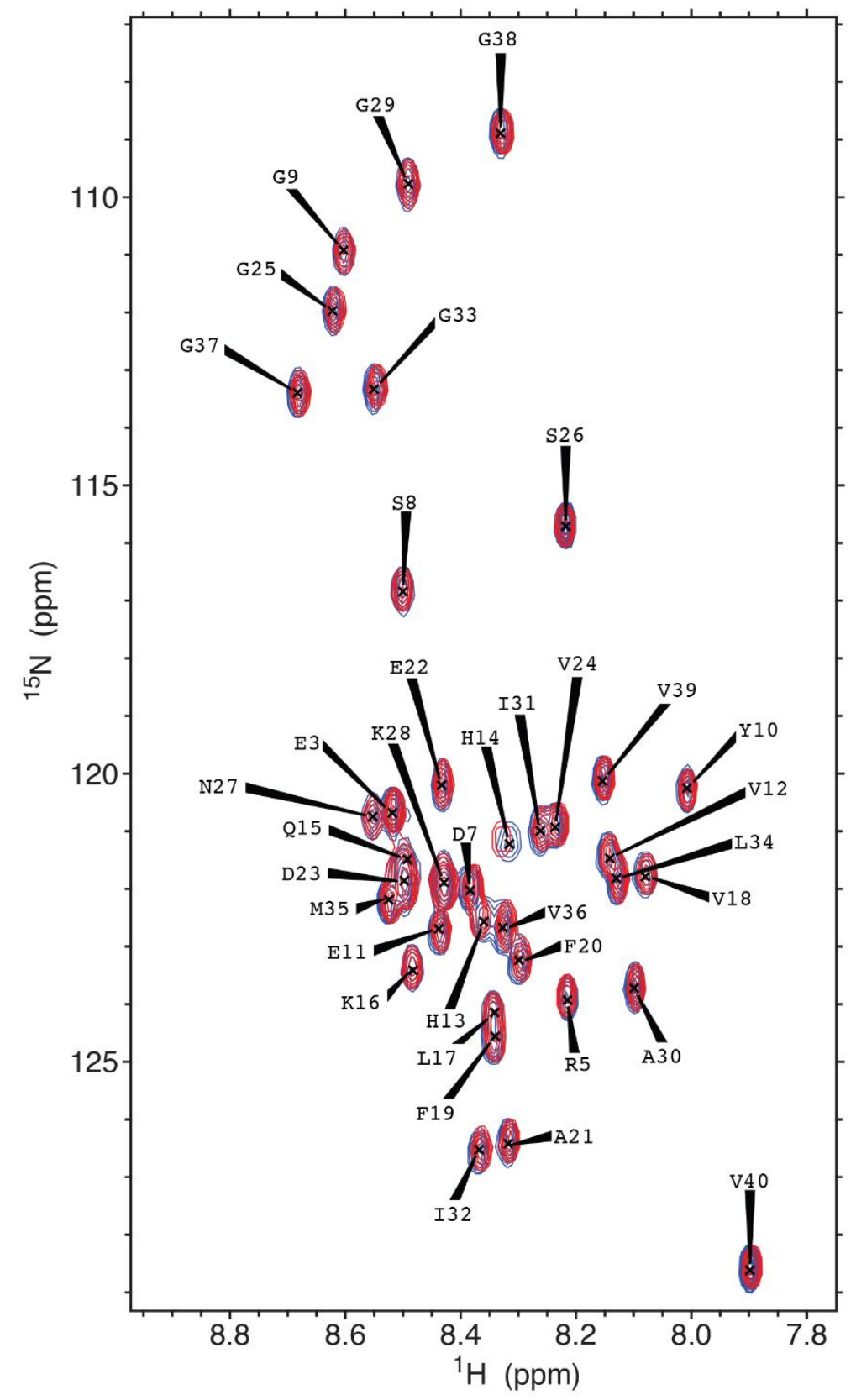

Figure S4: Two-dimensional ${ }^{1} \mathrm{H},{ }^{15} \mathrm{~N}-\mathrm{HSQC}$ NMR spectra of ${ }^{15} \mathrm{~N}$-labeled $\mathrm{A} \beta[1-40]$ in HFIP/phosphate buffer $\mathrm{pH} 7.2$ in the absence (red) and presence of (blue) 2-mM (+)MK-801. For clarity, spectra at intervening concentrations, which were recorded, are not shown. 


\section{REFERENCES}

[1] Ryan, T. M., Caine, J., Mertens, H. D., Kirby, N., Nigro, J., Breheney, K., Waddington, L. J., Streltsov, V. A., Curtain, C., Masters, C. L., and Roberts, B. R. (2013) Ammonium hydroxide treatment of Abeta produces an aggregate free solution suitable for biophysical and cell culture characterization, PeerJ 1, e73.

[2] Acerbo, A. S., Cook, M. J., and Gillilan, R. E. (2015) Upgrade of MacCHESS facility for Xray scattering of biological macromolecules in solution, J Synchrotron Radiat 22, 180186.

[3] Skou, S., Gillilan, R. E., and Ando, N. (2014) Synchrotron-based small-angle X-ray scattering of proteins in solution, Nature protocols 9, 1727-1739.

[4] Franke, D., Jeffries, C. M., and Svergun, D. I. (2015) Correlation Map, a goodness-of-fit test for one-dimensional X-ray scattering spectra, Nat Methods 12, 419-422.

[5] Hopkins, J. B., Gillilan, R. E., and Skou, S. (2017) BioXTAS RAW: improvements to a free open-source program for small-angle X-ray scattering data reduction and analysis, $J$ Appl Crystallogr 50, 1545-1553.

[6] Franke, D., Petoukhov, M. V., Konarev, P. V., Panjkovich, A., Tuukkanen, A., Mertens, H. D. T., Kikhney, A. G., Hajizadeh, N. R., Franklin, J. M., Jeffries, C. M., and Svergun, D. I. (2017) ATSAS 2.8: a comprehensive data analysis suite for small-angle scattering from macromolecular solutions, J Appl Crystallogr 50, 1212-1225.

[7] Millhauser, G. L., and Oswald, R. E. (1988) A reevaluation of the mathematical models for simulating single-channel and whole-cell ionic currents, Synapse 2, 97-103.

[8] MATLAB. (2018) version 9.5 (R2018b), Natick, Massachusetts: The Mathworks, Inc.

[9] Press, W. H., Flannery, B. P., Teukolsky, S. A., and Vetterling, W. T. (1986) Numerical 
recipes: The art of scientific computing, Cambridge University Press, New York. 